\title{
Corynebacterium auris
}

National Cancer Institute

\section{Source}

National Cancer Institute. Corynebacterium auris. NCI Thesaurus. Code C86310.

A species of aerobic or facultatively anaerobic, Gram-positive, coryneform, pleomorphic bacilli assigned to the phylum Actinobacteria. This species is nonmotile, non-spore forming, catalase positive, oxidase negative, pyrazinamidase positive, does not reduce nitrate, negative for urease, ferments glucose, but does not hydrolyze esculin. C. auris is associated with ear infections in the young er population. 